Review

\title{
Generation of Supramolecular Chirality around Twofold Rotational or Helical Axes in Crystalline Assemblies of Achiral Components
}

\section{Mikiji Miyata $^{1, *}$, Norimitsu Tohnai ${ }^{2}$, Ichiro Hisaki ${ }^{2}$ and Toshiyuki Sasaki ${ }^{3}$}

1 The Institute of Scientific and Industrial Research, Osaka University, Ibaraki, Osaka 567-0047, Japan

2 Department of Material and Life Science, Graduate School of Engineering, Osaka University, Suita, Osaka 565-0871, Japan; E-Mails: tohnai@mls.eng.osaka-u.ac.jp (N.T.);

hisaki@mls.eng.osaka-u.ac.jp (I.H.)

3 Department of Chemistry and Biology, Graduate School of Science and Engineering, Ehime University, Matsuyama, Ehime 790-8577, Japan; E-Mail: sasaki.toshiyuki.ag@ehime-u.ac.jp

* Author to whom correspondence should be addressed; E-Mail: miyata@mls.eng.osaka-u.ac.jp; Tel.: +81-6-6879-8496; Fax: +81-6-6879-8499.

Academic Editor: Rui Tamura

Received: 29 September 2015 / Accepted: 14 October 2015 / Published: 21 October 2015

\begin{abstract}
A multi-point approximation method clarifies supramolecular chirality of twofold rotational or helical assemblies as well as bundles of the one-dimensional (1D) assemblies. While one-point approximation of materials claims no chirality generation of such assemblies, multi-point approximations do claim possible generation in the $1 \mathrm{D}$ assemblies of bars and plates. Such chirality derives from deformations toward three-axial directions around the helical axes. The chiral columns are bundled in chiral ways through symmetry operations. The preferable right- or left-handed columns are bundled together to yield chiral crystals with right- or left-handedness, respectively, indicating that twofold helix symmetry operations cause chiral crystals composed of achiral components via a three-stepwise and three-directional process.
\end{abstract}

Keywords: supramolecular assemblies; supramolecular chirality; twofold helix; multi-point approximation; chirality generation; achiral components; columnar bundles; chiral crystals; handedness determination 


\section{Introduction}

Since the discovery of cholic acid inclusion compounds [1-3], our research has been devoted to supramolecular chirality in crystals of chiral molecules, such as steroids [4-10] and alkaloids [11-18]. The fascinating relations between molecules and their assemblies gradually directed us toward achiral molecules. In 2006, we encountered the fact that a twofold helical (21) assembly of benzene molecules in cholic acid channels (Figure 1a) is similar to stairs in daily life (Figure 1b) [19].

The evoking idea was that even achiral groups and molecules can afford twofold helical assemblies with supramolecular chirality [20,21]. However, every textbook for crystallography has the following content; both clockwise and anti-clockwise rotations by $180^{\circ}$ yield identical assemblies without handedness [22-24]. In fact, one can find only a single notation (21) for twofold helices (Figure 1c), in contrast to a pair of notations ( 31 and 32 ) for threefold helices (Figure 1d). Such a discrepancy between the fact and the theory prompted us to reconsider a root of molecular and supramolecular chirality, facing with the discrepancy which lies between twofold rotation operations in space groups and chirality generation in space geometry. Solution of the discrepancy exposed chirality in crystals and opened a new frontier in supramolecular chirality (Figure 2) [25-27].

(a)

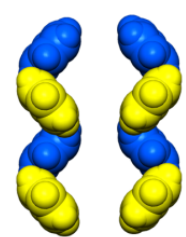

(b)

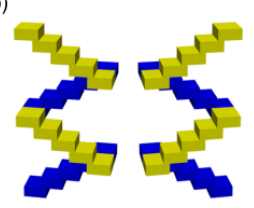

(c)
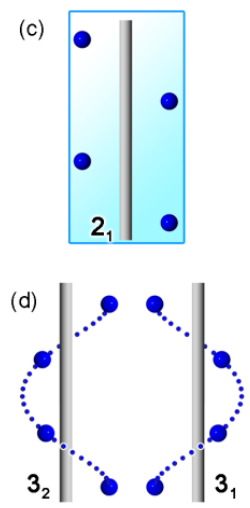

(e)
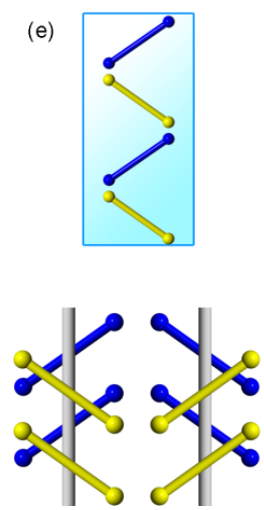

(f)
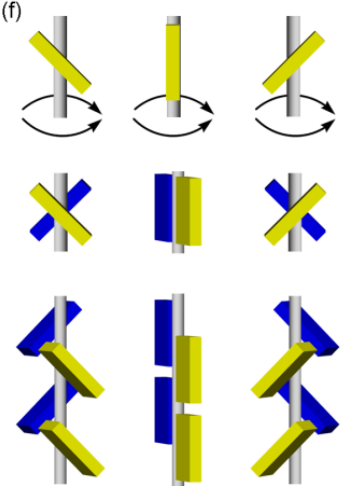

Figure 1. Twofold helical assembly of benzene molecules (a), stairs in daily life (b), twofold helix of points without handedness $\left(2_{1}\right)(\mathbf{c})$, threefold helix of points with right-handedness $\left(3_{1}\right)$ and left-handedness $\left(3_{2}\right)(\mathbf{d})$; chirality generation of $2{ }_{1}$ assemblies of bars (e) and twofold rotational and helical assemblies of tilt bars around an axis, where the symmetry operations have nothing to do with chirality generation (f).

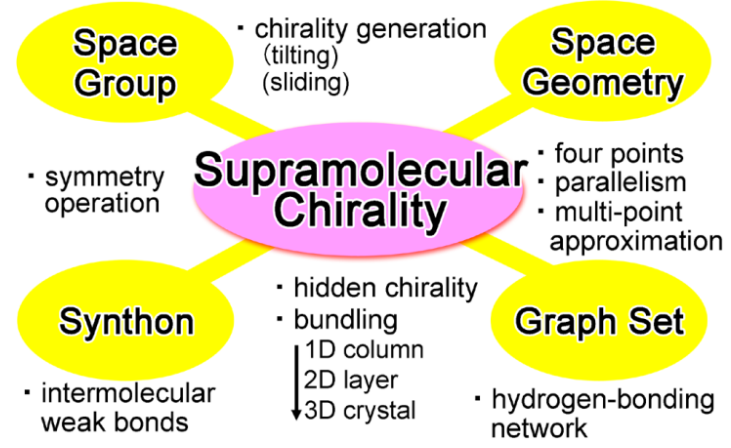

Figure 2. Research for exposing hidden supramolecular chirality in crystals on the basis of space geometry, space groups, supramolecular synthons, graph sets, and so on. 
This article deals with a geometric elucidation for generating supramolecular chirality in twofold helical assemblies of achiral components. The first section describes chirality generation of assemblies of bars and plates (Figure 1e) as well as tilt-chirality around an axis (Figure 1f) by multi-point approximation methods. The solution of the discrepancy proved generation of chiral assemblies from achiral components. Subsequently, we focus hitherto overlooked supramolecular chirality of twofold rotational or helical (21) molecular assemblies and their bundles in organic crystals. The resultant geometric method for constructing crystals is linked with the crystallographic space groups [22-24], supramolecular synthons [28], weak non-covalent interactions [29-31], and graph sets [32,33]. The final part is briefly concerned with a linkage between molecular and supramolecular chirality in the hierarchical structure of universe.

\section{Hitherto Overlooked Supramolecular Chirality in Organic Crystals}

\subsection{Supramolecular Chirality in Deformed Assemblies}

Molecular chirality has its origin in tetrahedral asymmetry, and diversifies into axial, facial, and helical asymmetry on the basis of strong covalent bonds. On the other hand, supramolecular chirality lies in molecular assemblies which are constructed by weak non-covalent bonds [34]. In principle, supramolecular assemblies are usually larger and more flexible than molecules. Therefore, slight deformations from a symmetric structure may construct supramolecular enantiomers, indicating that supramolecular chirality composed of achiral molecules can spread more comprehensively than molecular chirality. For simplicity, Figure 3a illustrates assemblies of achiral substances, such as two bars, two plates, a tetrahedron, and a cube.

Continuous helices of macromolecules, such as DNAs and proteins, have right- or left-handedness at a glance, and the same goes for threefold discontinuous helices. In contrast, twofold discontinuous helices involving rotations by $180^{\circ}$ have been left unsolved regarding chirality generation (Figure 3b). In general, slight steric differences are dynamically lost in solution state, but are fixed in solid states, particularly in crystals as a supramolecular entity [35]. Current computer graphics using crystal data enables us to precisely measure various slight deformations from a symmetric structure, namely, various locational differences between and/or among molecules. Nonetheless, supramolecular chirality in twofold discontinuous helices has not been discussed owing to the following mysterious reason.

(a)

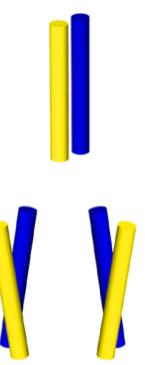

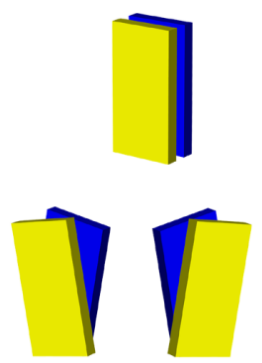

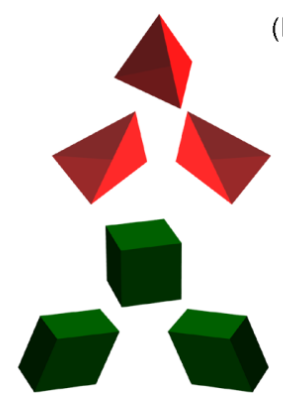

(b)

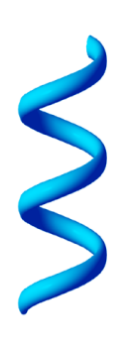

(c)
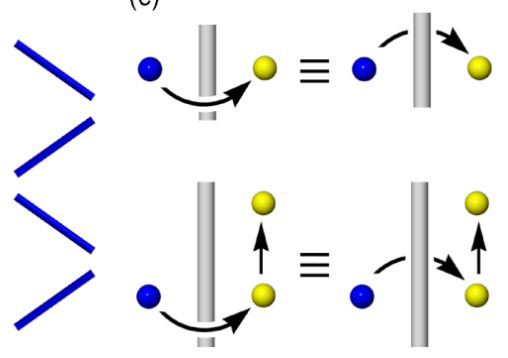

Figure 3. Generation of supramolecular enantiomers of two bars, two plates, a tetrahedron and a cube by slight deformations (a), chirality discrimination of continuous and discontinuous helices (b), and no generation of chirality by clockwise or anti-clockwise rotations by $180^{\circ}(\mathbf{c})$. 


\subsection{Conventional Idea for Chirality Generation in Major Crystals Involving $2{ }_{1}$ Helices}

The crystallography bases space group theory that crystalline assemblies are formed by various combinations of symmetry operations, such as inversion, rotation, and translation [22-24]. It is mathematically known that a twofold rotation operation by $180^{\circ}$ around an axis yields an identical assembly through both clockwise and anti-clockwise rotations. This links with the common belief that a twofold screw (or helix) (21) operation, which consists of both rotations by $180^{\circ}$ and translations by half of a pitch along a helical axis, yields an identical 21 assembly (Figure 3c). Consequently, the 21 assembly of achiral molecules does not exhibit supramolecular chirality, while that of chiral molecules exhibit it. As a result, supramolecular chirality of 21 assemblies composed of achiral molecules has been overlooked since the establishment of the crystallography in the first half of the last century [36-39].

Furthermore, it is well known that a majority of crystals contain the 21 operations [40]. In fact, the Cambridge Structural Database (CSD) includes about seven hundred thousand of crystal data, and remarkably over $70 \%$ of organic crystals involve the 21 operations [41]. Moreover, about $10 \%$ belong to chiral crystals composed of achiral molecules [36-39]. These facts indicate that it still remains open to interpret a mechanism for generating supramolecular chirality through the 21 operation. This mechanism may be closely related to that for enantiomeric resolution which has been continuously studied since the Pasteur's discovery [42].

\section{Chirality Generation from a Geometric Viewpoint}

Based on 3D space geometry, we present chirality generation in assemblies of points, lines, and planes [25-27]. Practically, instead of them, we can often use balls, bars, and plates, supposing achiral organic molecules which are contacted and do not penetrate each other.

\subsection{Four-point Assemblies and Their Handedness}

First of all, we consider the simplest assembly of points according to 3D space geometry (Figure 4a). A two- or three-point assembly makes a line or a plane without chirality, respectively. The fourth point may be laid on the plane without generating chirality, or separated from the mirror plane in accompany with chirality generation. This means that the four-point assembly is either non-enantiomeric or enantiomeric. Naturally, the four points can be deformed much more freely than the covalently bonded molecules. In this manner, the four-point assemblies serve as the simplest basis for chirality generation in $3 \mathrm{D}$ space.

Right- or left-handedness of the four-point chirality can be determined according to the following sequence rule (Figure 4b). The three points on the mirror plane (I, II, III) and the separating fourth point (IV or IV') are numbered with the predominant order; I > II > III > IV (IV'). As viewed from one side of the plane, the fourth point is placed in another side. When the points I, II, III are aligned clockwise or anti-clockwise, the assembly can be termed as right- or left-handed, respectively. This is similar to the well-known CIP rule for determining handedness in molecular chirality [43], and is consistent with the three-axial coordination system when the point I is located at the origin (Figure 4c). Conveniently, we can judge the handedness by using three fingers of thumb, index finger, and middle finger (Figure 4d). 
Such a consistency of molecular and supramolecular chirality permits us to designate the right- or left-handed supramolecular assembly as ${ }^{\sup } R$ or ${ }^{s u p} S$, respectively.

(a)

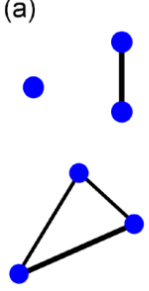

(b)

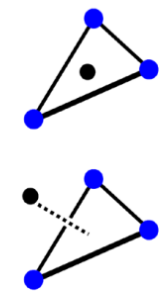

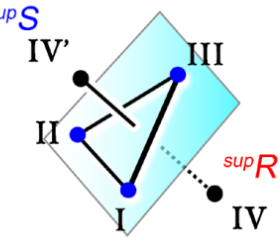

(c)

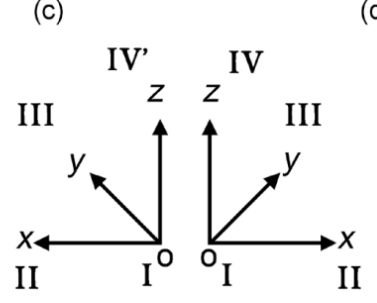

(d)

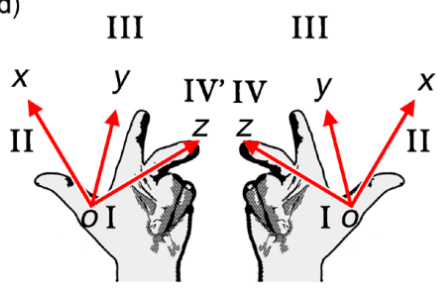

Figure 4. Chirality generation of four points (a); handedness determination of four points with a sequence order (b); right- or left-handed coordination system (c); and handedness expression by three fingers and base of right or left hand in accompany with the corresponding $x, y, z$-axes as well as an origin (d).

\subsection{Multi-point Approximation and Edge-view Methods for Bars and Plates}

Geometrically, points are extended to lines. Imaging achiral organic molecules, we can use simple balls and bars. This replacement induced us to employ a multi-point approximation method for bars, which suits for a linkage with the four-point chirality. For simplicity, the ball and bar are treated as one- and two-point approximations, respectively. Figure 5a depicts assemblies of two identical balls and bars. Two balls do not make an enantiomeric assembly, but the two bars make either a non-enantiomeric or enantiomeric assembly. This is because the two-point approximation method allows the two bars to have the four points necessary for chirality generation. The two bars align in parallel or non-parallel on a single plane to yield the four points without generating chirality, while they intersect in non-parallel to yield the four points suitable for generating chirality. Giving a repeated sequence of the two bars with the predominant order (I > II > III > IV (IV')), their handedness $\left({ }^{\sup } R\right.$ or ${ }^{\sup } S$ ) can be determined.

Next, the lines are extended to the planes or plates (Figure 5b). Three-point approximation method allows two plates to have six points which may yield diverse arrangements in 3D space. For simplicity, a plate can be treated as a line which functions as an edge-view of the plate, and the line is termed as e-line hereafter. Such e-lines of two identical plates enable us to briefly discriminate a non-enantiomeric arrangement in parallel from an enantiomeric one in non-parallel. Assuming a repeated sequence of the two e-lines $(\mathrm{I}>\mathrm{II}>\mathrm{III}>\mathrm{IV}(\mathrm{IV}))$, their handedness $\left({ }^{\sup } R\right.$ or $\left.{ }^{\sup } S\right)$ can be determined, as in the case of the two bars.

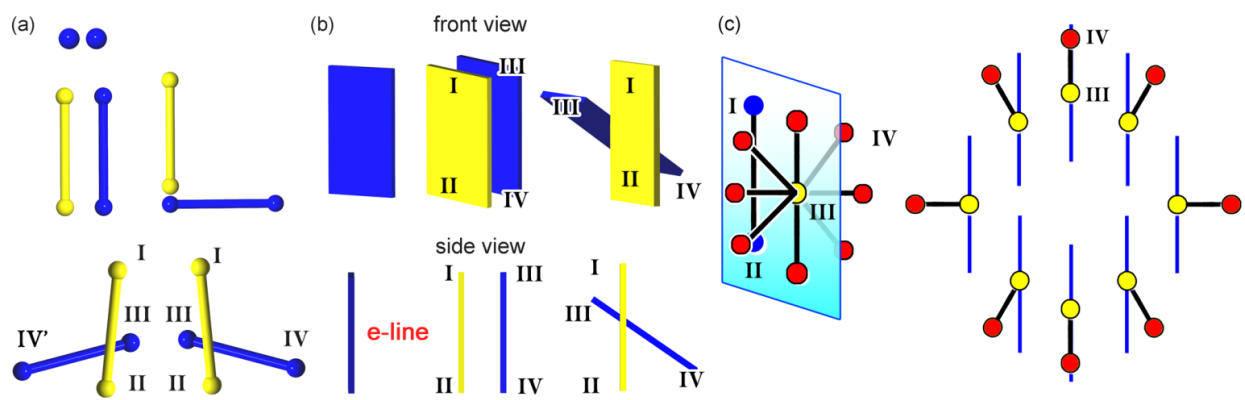

Figure 5. Chirality generation of two balls and two bars (a); two plates with e-lines as edge-views (b); as well as a bar with parallel, vertical, or tilt alignment along a virtual axis $(\mathbf{c})$. 


\subsection{Tilt-chirality around a Virtual Axis}

The four-point chirality is based on a mirror plane with the three points. This induced the idea that two points among the three points function as a virtual axis (Figure 5c). Namely, the e-line involving the mirror becomes the virtual axis. Along this axis, a bar takes various alignments, which are classified into three categories; parallel, perpendicular, and tilt. As viewed from outside, we recognize a supramolecular enantiomer of the bars with right- or left-tilt alignment along the axis, corresponding to right- or left-handedness $\left({ }^{\sup } R\right.$ or $\left.{ }^{\sup } S\right)$, respectively. This is termed as tilt-chirality around a virtual axis. It is important that this tilt-chirality emerges without the following symmetry operations.

\section{Chirality Generation by Symmetry Operations}

The point (or ball), line (or bar) and plane (or plate) in geometry are linked to symmetry operations, such as rotation, screw (or helix), and translation in crystallography. The operations change the virtual axis into a real rotational or helical axis, resulting in columnar assemblies.

\subsection{Rotation Operation}

Twofold rotation operation affords a twofold rotational assembly of two identical molecules along a twofold rotation axis. Figure 6 exemplifies two identical bars and plates. The bars and plates placed on a single plane generate no chirality (Figure 6a,b), but the intersecting ones generate it (Figure 6c,d). Namely, the resulting assemblies are either non-enantiomeric or enantiomeric. This simple fact has been overlooked, causing the conventional idea that twofold rotations by $180^{\circ}$ generate no chirality (Figure $3 \mathrm{c}$ ). We should focus attention on tilt-chirality around a virtual axis (Figures $1 \mathrm{f}$ and $5 \mathrm{c}$ ). In other words, clockwise and anti-clockwise twofold rotations by $180^{\circ}$ have nothing to do with chirality generation. At last, the discrepancy regarding chirality generation through twofold rotations was solved.

(a) II'

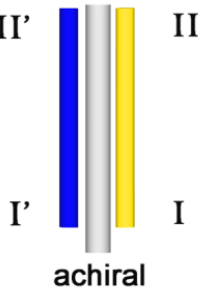

(b)

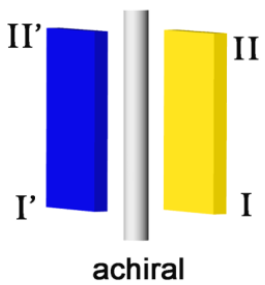

(c)

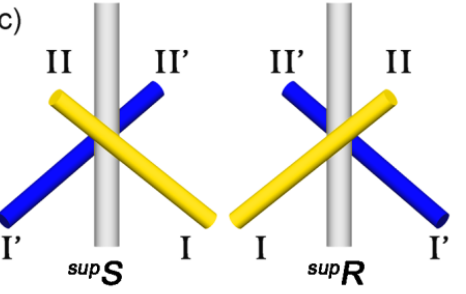

(d)

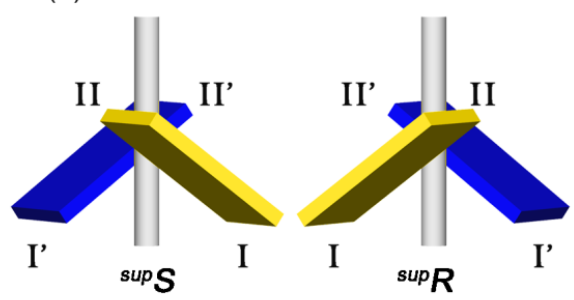

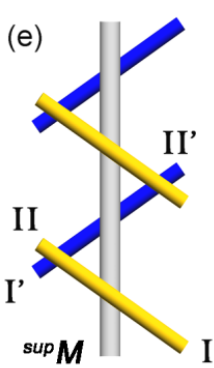
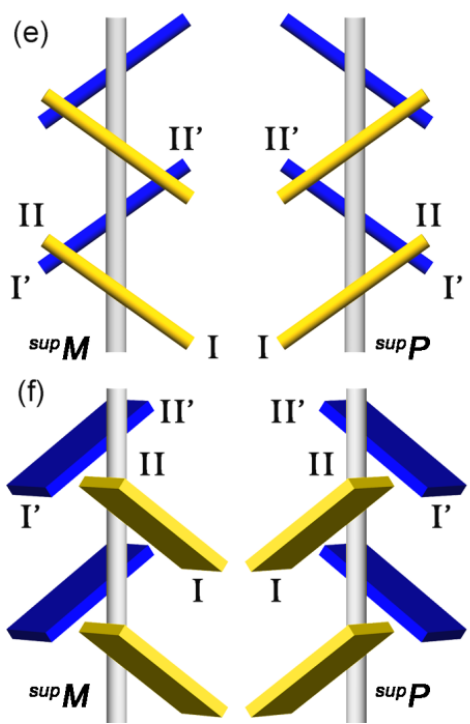

Figure 6. Generation of supramolecular tilt-chirality along a rotational or helical axis. Achiral assembly, chiral twofold rotation assembly, chiral twofold helix (21) assembly of bars (a, c, and e) and plates (b, d, and $\mathbf{f})$. 
As viewed from outside of the axis, the intersecting bars are right-tilt or left-tilt along the axis, defining right- or left-handed assemblies $\left({ }^{s u p} R\right.$ or $\left.{ }^{\sup } S\right)$, respectively. This is because the two-point approximation method enables us to assign a repeated sequence (I > II > I' > II'). Regarding the plates, the intersecting bars are treated as e-lines, and are extended to two non-parallel and tilt plates. Like the bars, the e-lines have a repeated sequence, so that we define right- or left-handed assemblies ${ }^{\sup R} R$ or

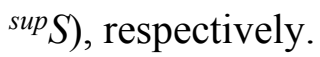

In this way, the twofold rotation operation can afford a supramolecular enantiomer of the assemblies on the basis of tilt-chirality along an axis. We termed such chirality as supramolecular tilt-chirality $[8,12,19,20]$. Similarly, the other three-, four-, and six-fold rotation operations in crystallography produce the corresponding assemblies of the identical bars and plates around their rotation axes. Each assembly exhibits chirality on the basis of tilt-chirality along each rotation axis, respectively.

\subsection{Helix (Screw) Operation}

A helix operation composes both rotation and translation operations. Therefore, the rotational assemblies are modified to helical ones through translation operation. For simplicity, Figure 6e exemplifies a $2{ }_{1}$ assembly of bars around a helical axis. The bars serve as e-lines and are extended to the helical assemblies of the plates (Figure 6f). The bars and plates on a single plane generate no chirality, but the intersecting bars and plates do it. As viewed from outside of the axis, the right- or left-tilt bars and e-lines along the helical axis correspond to right- or left-handed helical assemblies ( ${ }^{\sup P} \mathrm{P}$ or ${ }^{\sup } M$ ), respectively, owing to spontaneous assignment of a tandemly-repeated sequence (I > II > I' > II'). As a result, the twofold helix $(21)$ operation can prepare a supramolecular enantiomer of the assemblies with supramolecular tilt-chirality, as in the case of the rotation operations.

Regarding the other helix operations, it has been conventionally considered that three-fold helix operation exhibits chirality with a pair of notations, 31 and 32 , for right- and left-handedness, respectively. Similarly, there exist supramolecular enantiomers of 41 and 43, 61 and 65, 62 and 64, compared to no pairs of $4_{2}$ and 63 . However, the above-mentioned mechanism for chirality generation indicates that the $2{ }_{1}, 42$, and 63 helical assemblies of bars and plates are either non-enantiomeric or enantiomeric, as in the case of two-, three-, four-, and six-fold rotational assemblies.

\subsection{Translation Operation and Others}

In the case of the 21 helix operation, a virtual axis functions as a rotation axis which is parallel to a translation axis. Geometrically, the translation axis may become non-parallel to the rotation axis, enabling to yield a supramolecular enantiomer of translational assemblies of the planes (Figure 7a). It is noteworthy that points and lines do not yield a supramolecular enantiomer of the translational assemblies, since they are located on a single plane. Handedness determination needs to discriminate front- and back-sides of the plane. For example, facial molecules with prochirality would become possible. The front-sides of the plates slide to right-to-top or left-to-bottom, resulting in a right-handed assembly, and vice versa. The other inversion operation of the plane produces a racemic assembly in anti-parallel alignment (Figure $7 b$ ). 
(a)

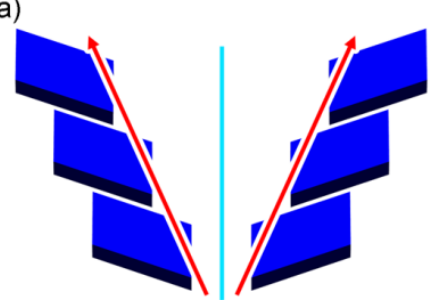

(b)

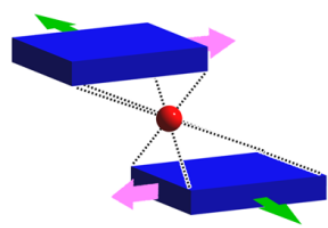

Figure 7. Chirality generation in translational assemblies of plates (a); and formation of achiral anti-parallel planes through inversion operation (b).

\subsection{New Idea for Chirality Generation through Twofold Rotations}

In this way, the conventional idea for rotations by $180^{\circ}$ must be changed to the new idea as follows (Figure 1f). The clockwise or anti-clockwise rotations around an axis have nothing to do with chirality generation, while tilt alignments of bars or plates along the axis cause supramolecular chirality. Their handedness is attributed to the right- or left-tilt alignment around the axis, in contrast to the CIP rules in molecular chirality.

\subsection{Supramolecular Chirality of $2{ }_{1}$ Helical Columns of Bars and Plates}

A continuous helix has three directions around a helical axis; right-and-left, in-and-out as well as up-and-down [25-27]. In the case of a 21 discontinuous helix, these directions are related to $x, y, z$-axes of three-axial coordination system, where the helical axis is located along $z$-axis (Figure 8a). We always observe any sliding along $x, y, z$-axes in the 21 columns of bars (Figure $8 \mathrm{~b}$ ) and plates (Figure 8c). Such deformations from symmetric structures can expose hitherto overlooked supramolecular chirality of the $2{ }_{1}$ helices. Remarkably, the up-and-down direction along the $z$-axis exhibits one mountain upside and the other valley downside, enabling to employ a graphical symbol with an attached arrow, like a continuous helix (Figure 8d).

(a)

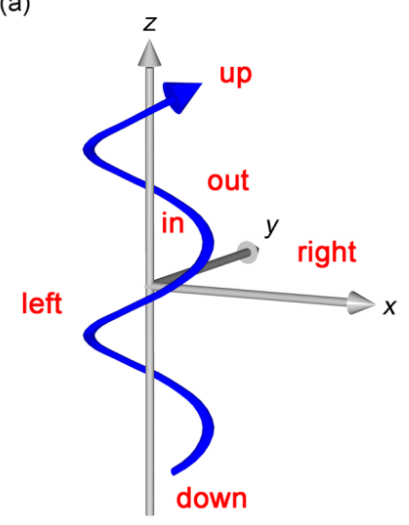

handedness: $P$ (b)

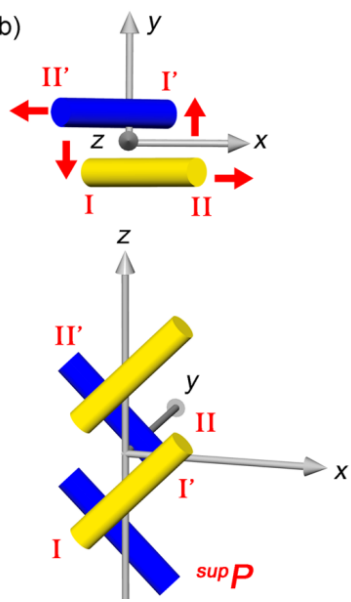

(c)

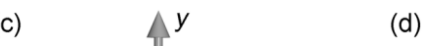

(d)

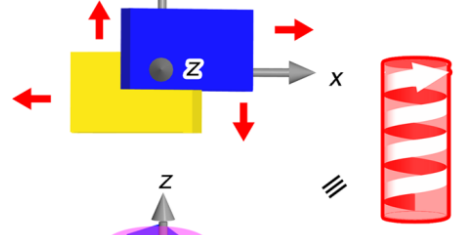

mountain-side

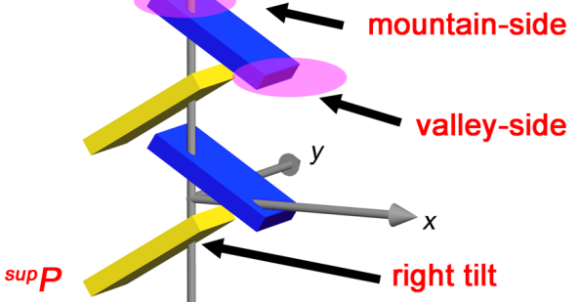

Figure 8. Chirality generation based on deformations. Continuous right-handed helix with directionality in accompany with an arrow and $x, y, z$-axes (a); deformations due to sliding along $x, y, z$-axes in discontinuous 21 assemblies of bars (b) and plates (c), and discriminable up-and-down direction owing to mountain- or valley-side in accompany with a graphical helix with an arrow (d). 


\section{Chirality Generation by Bundling of 21 Helical Columns}

According to crystallography, combinations of symmetry operations are classified into 230 space groups [22-24]. Among them, we focus on major space groups involving $2{ }_{1}$ operations which produce various 21 columnar assemblies.

\subsection{Bundles of the Preferable 21 Columns}

The International Tables for Crystallography employs only one symbol for 21 symmetry operation, as exemplified in a unit cell for space group $P 2{ }_{1}$ (Figure 9a) [22]. However, in the case of bars and plates, the $21_{1}$ operation can bring about different $2{ }_{1}$ columnar assemblies with deformations along three axes, prompting us to modify the conventional symbol (Figure 9b) [25-27]. Thus, the right-and-left direction is discriminated with an external curve, while the up-and-down direction is done with a black circle and an $x$ mark in the center. Figure $9 \mathrm{c}$ displays four different $2{ }_{1}$ columns in space group $P 21$, where right- or left-handed columns are arbitrarily selected. The existence of various 21 columns in the unit cell insisted us to select a preferable 21 column (Figure 9d) [26,27] The preferable column can be evaluated by the supramolecular synthons [28] and intermolecular weak bonds [29-31], enabling to draw bundles of the columns by using the graphical symbols in Figure $8 \mathrm{~d}$. Figure 9e exemplifies a bundle of the right-handed preferable columns in space group $P 2{ }_{1}$. In addition, symmetry operations necessary for the bundle formation are inserted between the preferable helices by using lines for $2_{1}$ helix operation as well as dots for inversion operation. Figure 10a-d show the bundles for $P 2_{1}, P 2{ }_{1}{ }_{1} 2_{1}, P 2_{1} / c, P b c a$, respectively.

(a)

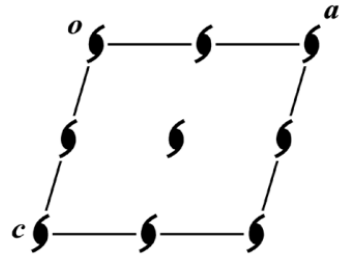

(b)

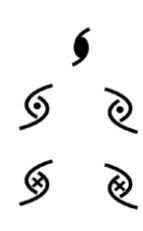

(c)

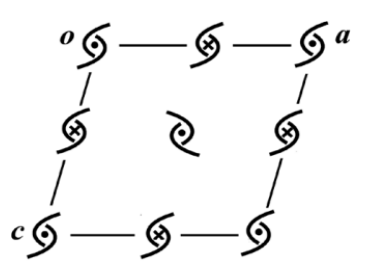

(d)

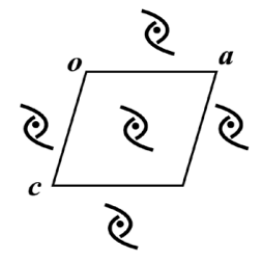

(e)

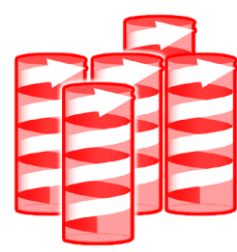

Figure 9. Representation of space group $P 21$. Symmetry operation with an identical symbol (a); modified symbols for right- and left-handedness as well as up-and-down direction (b); bundle of different 21 columns with the modified symbols (c); bundle of the preferred 21 columns with the modified symbols (d) and with graphical helices (e).

(a)

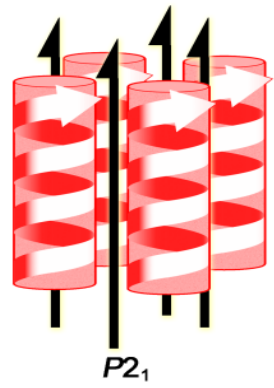

(b)

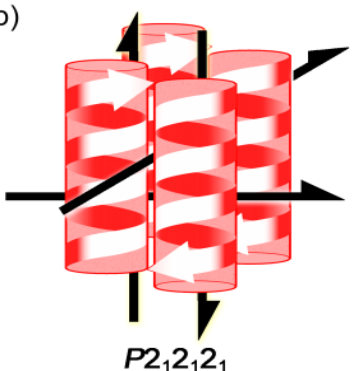

(c)

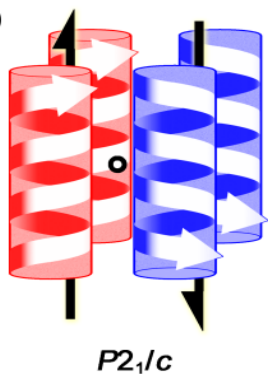

(d)

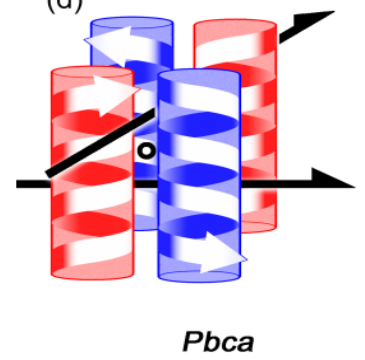

Figure 10. Bundles involving symmetry operations between the preferable columns for space groups, $P 2{ }_{1}$ (a), $P 2{ }_{1} 2{ }_{1}{ }_{1}$ (b), $P 2{ }_{1} / c$ (c), and $P b c a$ (d). The lines and dots designate 21 helix and inversion operations, respectively. 


\subsection{Chirality Generation in Bottom-up Construction toward Crystals from Achiral Molecules}

From a comparison of these figures, we proposed a bottom-up method for bundling the preferable column, termed as three-stepwise and three-directional process [44,45]. The first step corresponds to formation of the preferable column along the $z$-axis. The second step lies in layer formation along the $y$-axis, and the third step consists of layer stacking along the $x$-axis. Such a bottom-up construction process can be linked with space groups as follows. Figure 11 displays the process for space groups involving the $2{ }_{1}$ assemblies; $P 2{ }_{1}, P 2{ }_{1}{ }_{1}{ }_{2}, P 2{ }_{1} / c$, and $P b c a$. The process clarifies chirality generation in each step. In the first step, chirality arises from a preferable $2{ }_{1}$ column with right- or left-handedness (Figure 11a). In the second step, chirality is derived from alignments of the columns. The right- or left-handed $2{ }_{1}$ columns alternatively align for space group $\mathrm{Pbca}$, while the one-handed 21 columns align in parallel in keeping with the identical up-and-down direction, resulting in chiral layers for space groups $P 21, P 2{ }_{12} 2_{1}$, and $P 2{ }_{1} / c$ (Figure 11b). In the third step, chirality is based on the layer-stacking. The achiral layers stack for $P b c a$, and the right- or left-handed layers alternatively stack for $P 2{ }_{1} / c$. In contrast, the one-handed layers stack in parallel for $P 2{ }_{1}$ (Figure 11c) and in anti-parallel for $P 2{ }_{1}{ }_{1}{ }_{1}$ (Figure 11d).
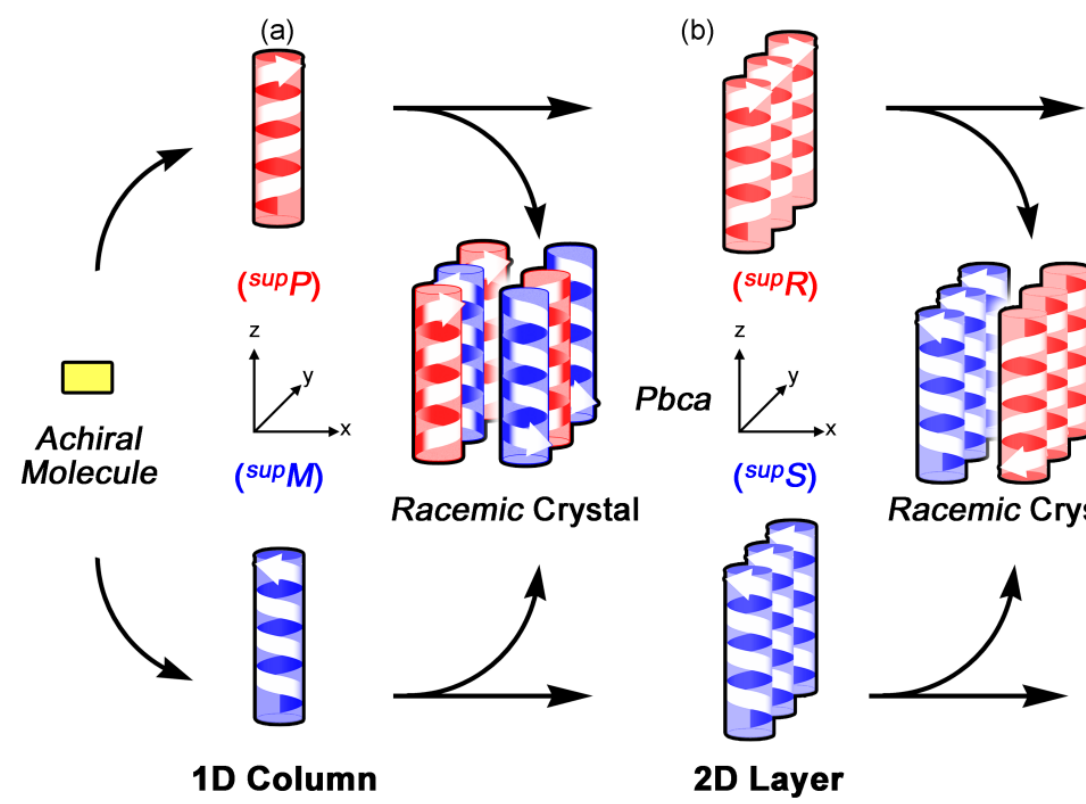

(c)

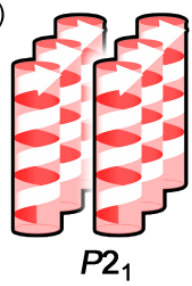

(d)

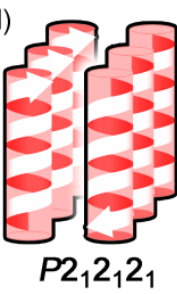

(supR)

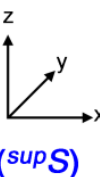

$P 2 / c$

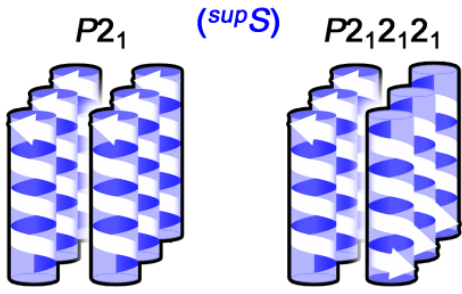

3D Crystal

Figure 11. The bottom-up construction process for space groups involving the $2{ }_{1}$ assemblies; $P 2{ }_{1}, P 2{ }_{1} 2_{2}{ }_{1}, P 21 / c$, and Pbca. Chiral columns composed of achiral molecules (a); chiral layers (b); chiral crystals by the layer stacking for $P 2{ }_{1}$ (c) and $P 2{ }_{1}{ }_{1} 2_{1}$ (d).

\subsection{Chiral Space Groups}

Sixty-five among 230 groups are known as the chiral Sohncke symmetry space groups [39]. When symmetry operations are discriminable in chirality by themselves, they hold a pair of notations. Namely, 22 groups involving 31 and 32,41 and 43,61 and 65,62 and 64 belong to chiral groups. On the other hand, the other 43 groups involving translation, 2, 3, 4, 6 rotations, as well as 21,42 , 63 helix operations yield either non-enantiomeric or enantiomeric assemblies as mentioned above. Therefore, the 43 groups belong to either chiral or achiral groups, meaning that achiral molecules have latent possibility to yield chiral crystals which belong to any of the Sohncke space groups. 


\section{Linkage between Molecular and Supramolecular Chirality}

Chirality generations through deformation of hydrogen-bonding networks are attractive. A wide variety of the networks are well-known, including 0D rings or cubics [46], 1D chains or columns [47], and 2D sheets [48]. Their prototypes have symmetric structures, which are deformed by slight differences of molecular structures [49]. The resulting supramolecular chirality insisted us to advance the graph sets analysis which was established for systematic designation of the hydrogen-bonding networks [32,33]. Alternatively, cubic clusters with triphenylmethyl (trityl) groups were found to become analogues of octacoordinated polyhedrons, such as triangular dodecahedron and trans-bicapped octahedron [50]. Interestingly, these polyhedrons exhibit flexibility and diversity of supramolecular chirality owing to deformation of the hydrogen-bonding networks.

A molecule with a single chiral element can yield an enantiomer of molecules with molecular chirality as well as a supramolecular enantiomer of molecular assemblies with supramolecular chirality. For example, a molecule with one stereogenic carbon $\left(R\right.$ or $S$ ) can yield an enantiomeric assembly ( ${ }^{\sup } R$ or $\left.{ }^{s u p} S\right)$, resulting in four combinations; $\left(R,{ }^{s u p} R\right),\left(R,{ }^{s u p} S\right),\left(S,{ }^{s u p} R\right)$, and $\left(S,{ }^{s u p} S\right)$. Regarding the 21 assemblies, such a linkage between molecular chirality $(R$ or $S)$ and supramolecular chirality ( ${ }^{\text {sup }} P$ or ${ }^{\sup } M$ ) has been confirmed by using organic salts of primary ammonium carboxylates [51] as well as 2-pyridyl alcohols [52]. Alternatively, the $2{ }_{1}$ assemblies of crystalline monomers such as benzoquinone methides undergo twofold helical polymerization [53]. Such a chirality linkage in organic crystals has been so far hidden in the hierarchical structures of universe [54,55].

\section{Conclusions and Outlook}

Eventually, we solved the overlooked problem of chirality generation through twofold rotations, leading to the idea that achiral molecules can create chiral $2{ }_{1}$ columns which are bundled via chiral bottom-up process to yield chiral crystals. It is attractive that each step of the process involves generation of supramolecular chirality, and that over $70 \%$ of the CSD involve the twofold helices. Hopefully, this article prompts to design achiral molecules suitable for preparing chiral crystals.

Fortunately, it has been confirmed that handedness determination methods are consistent among molecules, supramolecules, and materials in daily life, based on 3D space geometry. This is favorable for seeking a chirality linkage among the hierarchical structures in universe [54,55], where supramolecular chirality in crystals are significant as one of the expression of molecular information $[3,4,8,10]$. The 21 helical columns seem to be analogous to 3D building-bricks in daily life, inducing a delightful research in crystal engineering toward creation of chiral materials, including organic, inorganic and polymeric compounds, starting from achiral components.

\section{Acknowledgments}

The authors sincerely thank our colleagues and students who have participated as coauthors. Continuing support for our research from MEXT is greatly acknowledged.

\section{Conflicts of Interest}

The authors declare no conflict of interest. 


\section{References}

1. Miyata, M.; Shibakami, M.; Goonewardena, W.; Takemoto, K. Inclusion compounds of cholic acid with a variety of organic substances. Chem. Lett. 1987, 16, 605-608.

2. Miki, K.; Masui, A.; Kasai, N.; Miyata, M.; Shibakami, M.; Takemoto, K. New channel-type inclusion compound of steroidal bile acid. Structure of a 1:1 complex between cholic acid and acetophenone. J. Am. Chem. Soc. 1988, 110, 6594-6596.

3. Miyata, M.; Shibakami, M.; Chirachanchai, S.; Takemoto, K.; Kasai, N.; Miki, K. Guest-responsive structural changes in cholic acid intercalation crystals. Nature 1990, 343, 446-447.

4. Miyata, M.; Sada, K. Deoxycholic Acid and Related Hosts. In Solid-State Supramolecular Chemistry, Comprehensive Supramolecular Chemistry; MacNicol, D.D., Toda, F., Bishop, R., Eds.; Pergamon: Oxford, UK, 1996; Volume 6, pp. 147-176.

5. Miyata, M.; Sada, K.; Yoswathananont, N. Deoxycholic, Cholic, and Apocholic Acids. In Encyclopedia of Supramolecular Chemistry; Atwood, J.L., Steed, J.W., Eds.; Marcel Dekker: New York, NY, USA, 2004; Volume 1, pp. 441-451.

6. Miyata, M.; Yoswathananont, N.; Nakano, K.; Sada, K. Separation of Isomers and Enantiomers by Bile Acid Derivatives. In Separation and Reaction in Organic Supramolecular Chemistry, Perspectives in Supramolecuar Chemistry; Toda, F., Bishop, R., Eds.; John Wiley \& Sons: England, UK, 2004; Volume 8, pp. 87-122.

7. Miyata, M.; Tohnai, N.; Hisaki, I. Supramolecular chirality in crystalline assemblies of bile acids and their derivatives; Three-axial, tilt, helical, and bundle chirality. Molecules 2007, 12, 1973-2000.

8. Miyata, M.; Tohnai, N.; Hisaki, I. Crystalline host-guest assemblies of steroidal and related molecules: Diversity, hierarchy, and supramolecular chirality. Acc. Chem. Res. 2007, 40, 694-702.

9. Nakano, K.; Aburaya, K.; Hisaki, I.; Tohnai, N.; Miyata, M. Flexible host frameworks with diverse cavities in inclusion crystals of bile acids and their derivatives. Chem. Rec. 2009, 9, 124-135.

10. Miyata, M.; Sada, K.; Nakano, K.; Tohnai, N. Inclusion Compounds of Steroidal Molecules: Molecular Information and Their Expression through Molecular Assemblies. In Bottom-Up Nanofabrication; Nalwa, H.S., Ariga, K., Eds.; American Scientific Publisher: Valencia, CA, USA, 2009; Volume 3, pp. 147-169.

11. Watabe, T.; Yoshikawa, D.; Hisaki, I.; Tohnai, N.; Miyata, M. Supramolecular chirality and isomerism in cinchonidine crystals: Hierarchical analysis on the basis of the asymmetric 21 helical columnar assembly. Chem. Lett. 2006, 35, 806-807.

12. Hisaki, I.; Watabe, T.; Kogami, Y.; Tohnai, N.; Miyata, M. $2{ }_{1}$ Helical assemblies of cinchona alkaloids in crystals: Definition of their handedness based on the molecular tilt. Chem. Lett. 2006, $35,1274-1275$.

13. Watabe, T.; Hisaki, I.; Tohnai, N.; Miyata, M. Four kinds of $2{ }_{1}$ helical assemblies with the molecular tilt as well as three-directional and facial chirality. Chem. Lett. 2007, 36, 234-235.

14. Watabe, T.; Kobayashi, K.; Hisaki, I.; Tohnai, N.; Miyata, M. Guest-induced supramolecular isomerism and chirality of brucine inclusion crystals with aliphatic alcohols: A hierarchical interpretation. Bull. Chem. Soc. Jap. 2007, 80, 464-475. 
15. Hisaki, I.; Shizuki, N.; Aburaya, K.; Katsuta, M.; Tohnai, N.; Miyata, M. Structures of brucinium cholate: Bile acid and strychinine derivatives meet in the crystals. Cryst. Growth Des. 2009, 9, 1280-1283.

16. Hisaki, I.; Shizuki, N.; Sasaki, T.; Ito, Y.; Tohnai, N.; Miyata, M. Handedness determination of 21 helical motifs and hierarchical analysis of crystal structures based on the motifs: The case of cinchona alkaloid derivatives. Cryst. Growth Des. 2010, 10, 5262-5269.

17. Sasaki, T.; Shizuki, N.; Hiraishi, E.; Tohnai, N.; Miyata, M. Construction of multi-component supramolecular architectures of bile acids and cinchona alkaloids through helical-pitch-synchronized crystallization. Org. Biomol. Chem. 2012, 10, 5985-5992.

18. Hisaki, I.; Hiraishi, E.; Sasaki, T.; Orita, H.; Tsuzuki, S.; Tohnai, N.; Miyata, M. Crystal structure of quinine: The effects of vinyl and methoxy groups on molecular assemblies of cinchona alkaloids cannot be ignored. Chem. Asian J. 2012, 7, 2607-2614.

19. Tanaka, A.; Hisaki, I.; Tohnai, N.; Miyata, M. Supramolecular tilt-chirality derived from symmetric benzene molecules: Handedness of the $2{ }_{1}$ helical assembly. Chem. Asian J. 2007, 2, 230-238.

20. Hisaki, I.; Tohnai, N.; Miyata, M. Supramolecular tilt chirality in crystals of steroids and alkaloids. Chirality 2008, 20, 330-336.

21. Hisaki, I.; Sasaki, T.; Sakaguchi, K.; Liu, W.T.; Tohnai, N.; Miyata, M. Right- and left-handedness of 21 symmetrical herringbone assemblies of benzene. Chem. Commun. 2012, 48, 2219-2221.

22. Koch, E.; Fisher, W.; Iler, U.M. International Tables for Crystallography, Vol. A, Space-Group Symmetry; Hahn, T., Ed.; Kluwer Academic Publishers: London, UK, 2002.

23. Glusker, J.P.; Trueblood, K.N. Crystal Structure Analysis: A Primer, 3rd ed.; Oxford University Press: Oxford, UK, 2010.

24. Bennett, D.W. Understanding Single-Crystal X-ray Crystallography; Wiley-VCH: Weinheim, Germany, 2010.

25. Hisaki, I.; Sasaki, T.; Tohnai, N.; Miyata, M. Supramolecular-tilt-chirality on twofold helical assemblies. Chem. Eur. J. 2012, 18, 10066-10073.

26. Hisaki, I.; Sasaki, T.; Tohnai, N.; Miyata, M. Multipoint approximation method for handedness determination of two-fold helical assemblies and their bundles. J. Syn. Org. Chem. Jpn. 2012, 70, 908-917.

27. Miyata, M.; Hisaki, I. Twofold Helical Molecular Assemblies in Organic Crystals: Chirality Generation and Handedness Determination. In Advances in Organic Crystal Chemistry; Tamura, R.; Miyata, M., Eds.; Springer Japan: Tokyo, Japan, 2015; Chapter 19, pp. 371-392.

28. Desiraju, G.R. Supramolecular synthons in crystal engineering-A new organic synthesis. Angew. Chem. Int. Ed. Engl. 1995, 34, 2311-2327.

29. Nishio, M.; Hirota, M.; Umezawa, Y. The CH/ Interaction-Evidence, Nature, and Consequences; Wiley-VCH: New York, NY, USA, 1998.

30. Desiraju, G.R.; Steiner, T. The Weak Hydrogen Bond in Structural Chemistry and Biology; Oxford University Press: Oxford, UK, 1999.

31. Gilla, G.; Gilli, P. The Nature of the Hydrogen Bond; Oxford University Press: Oxford, UK, 2009.

32. Etter, M.C. Encoding and decoding hydrogen-bond patterns of organic compounds. Acc. Chem. Res. 1990, 23, 120-126. 
33. Grell, J.; Bernstein, J.; Tinhofer, G. Graph-set analysis of hydrogen-bond patterns: Some mathematical concepts. Acta Crystallogr. B 1999, 55, 1030-1043.

34. Lehn, J.-M. Supramolecular Chemistry: Concepts and Perspectives; Wiley-VCH: Weinheim, Germany, 1995.

35. Desiraju, G.R. The Crystal as a Supramolecular Entity; John Wiley \& Sons: Chichester, UK, 1995.

36. Matsuura, T.; Koshima, H. Chiral crystallization of achiral organic compounds - Generation of chirality without chiral environment. Part 1. J. Syn. Org. Chem. Jpn. 1998, 29, 268-279.

37. Matsuura, T.; Koshima, H. Chiral crystallization of achiral organic compounds - Generation of chirality without chiral environment. Part 2. J. Syn. Org. Chem. Jpn. 1998, 29, 466-477.

38. Matsuura, T.; Koshima, H. Introduction to chiral crystallization of achiral organic compounds: Spontaneous generation of chirality. J. Photochem. Photobiol. C Photo. Rev. 2005, 6, 7-24.

39. Dryzun, C.; Avnir, D. On the abundance of chiral crystals. Chem. Commun. 2012, 48, 5874-5876.

40. Kitaigorodskii, A.I. Molecular Crystals and Molecules; Academic Press: New York, NY, USA, 1973.

41. Cambridge Structural Database. Available online: http://www.ccdc.cam.ac.uk/products/csd/statistics/ (accessed on 16 February 2015).

42. Jacques, J.; Collet, A.; Wilen, S.H. Enantiomers, Racemates, and Resolutions; Krieger: Malabar, FL, USA, 1991.

43. Cahn, R.S.; Ingold, C.K.; Prelog, V. Specification of molecular chirality. Angew. Chem. Int. Ed. 1966, 5, 385-415.

44. Sasaki, T.; Hisaki, I.; Tsuzuki, S.; Tohnai, N.; Miyata, M. Halogen bond effect on bundling of hydrogen bonded 2-fold helical columns. CrystEngComm 2012, 14, 5749-5752.

45. Sasaki, T.; Ida, Y.; Tanaka, A.; Hisaki, I.; Tohnai, N.; Miyata, M. Chiral crystallization by non-parallel face contacts on the basis of three-axially asymmetric twofold helices. CrystEngComm 2013, 15, 8237-8240.

46. Yuge, T.; Tohnai, N.; Fukuda, T.; Hisaki, I.; Miyata, M. Topological study of pseudo-cubic hydrogen-bond networks in a binary system composed of primary ammonium carboxylates: An analog of an ice cube. Chem. Eur. J. 2007, 13, 4163-4168.

47. Yuge, T.; Sakai, T.; Kai, N.; Hisaki, I.; Miyata, M.; Tohnai, N. Topological classification and supramolecular chirality of 21-helical ladder-type hydrogen-bond networks composed of primary ammonium carboxylates: Bundle control in 21-helical assemblies. Chem. Eur. J. 2008, 14, 2984-2993.

48. Tanaka, A.; Inoue, K.; Hisaki, I.; Tohnai, N.; Miyata, M.; Matsumoto, A. supramolecular chirality in layered crystals of achiral ammonium salts and fatty acids: A hierarchical interpretation. Angew. Chem. Int. Ed. 2006, 45, 4142-4145.

49. Sasaki, T.; Ida, Y.; Hisaki, I.; Yuge, T.; Uchida, Y.; Tohnai, N.; Miyata, M. Characterization of supramolecular hidden chirality of hydrogen-bonded networks by advanced graph set analysis. Chem. Eur. J. 2014, 20, 2478-2487.

50. Sasaki, T.; Ida, Y.; Yuge, T.; Yamamoto, A.; Hisaki, I.; Tohnai, N.; Miyata, M. Chirality generation in supramolecular clusters: Analogues of octacoordinated polyhedrons. Cryst. Growth Des. 2015, $15,658-665$. 
51. Sasaki, T.; Hisaki, I.; Miyano, N.; Tohnai, N.; Morimoto, K.; Sato, H.; Tsuzuki, S.; Miyata, M. Linkage control between molecular and supramolecular chirality in 21-helical hydrogen-bonded networks using achiral components. Nat. Commun. 2013, 4, doi:10.1038/ncomms2756.

52. Tsang, M.Y.; Salvo, F.D.; Teixidor, F.; Vinas, C.; Planas, J.G.; Choquesillo-Lazarte, D.; Vanthuyne, N. Is molecular chirality connected to supramolecular chirality? The particular case of chiral 2-pyridyl alcohols. Cryst. Growth Des. 2015, 15, 935-945.

53. Itoh, T.; Tachino, K.; Akira, N.; Uno, T.; Kubo, M.; Tohnai, N.; Miyata, M. Twofold helical polymerization: Thermal solid-state polymerization of 7-cyano-7-(2'-haloethoxycarbonyl)-1,4benzoquinone methides. Macomolecules 2015, 48, 2935-2947.

54. Gardner, M. The New Ambidextrous Universe; W.H. Freeman \& Company: New York, NY, USA, 1999.

55. Hegstrom, R.A.; Kondepudi, D.K. The handedness of the universe. Sci. Am. 1990, 262, 108-115.

(C) 2015 by the authors; licensee MDPI, Basel, Switzerland. This article is an open access article distributed under the terms and conditions of the Creative Commons Attribution license (http://creativecommons.org/licenses/by/4.0/). 